\title{
PET Mapping of Neurofunctional Changes in a Posttraumatic Stress Disorder Model
}

\author{
Yunqi Zhu*1-4, Ruili Du*1-4, Yuankai Zhu ${ }^{1-4}$, Yehua Shen ${ }^{1-4}$, Kai Zhang ${ }^{1-4}$, Yao Chen ${ }^{1-4}$, Fahuan Song ${ }^{1-4}$, \\ Shuang $\mathrm{Wu}^{1-4}$, Hong Zhang ${ }^{1-5}$, and Mei Tian ${ }^{1-5}$ \\ ${ }^{I}$ Department of Nuclear Medicine, The Second Hospital of Zhejiang University School of Medicine, Hangzhou, China; ${ }^{2}$ Zhejiang \\ University Medical PET Center, Hangzhou, China; ${ }^{3}$ Key Laboratory of Medical Molecular Imaging of Zhejiang Province, Hangzhou, \\ China; ${ }^{4}$ Institute of Nuclear Medicine and Molecular Imaging, Zhejiang University, Hangzhou, China; and ${ }^{5}$ Collaborative Innovation \\ Center for Brain Science, Fudan University, Shanghai, China
}

Posttraumatic stress disorder (PTSD) is an anxiety disorder that occurs after exposure to a traumatic event. This study aimed to investigate the neurobiologic changes before and after exposure-based therapy by PET in a rat model of PTSD. Methods: Serial ${ }^{18} \mathrm{~F}-\mathrm{FDG}$ PET imaging studies were performed under the control (tone presentation), fear-conditioning, and extinction retrieval phases. Neuroactivity marker c-Fos protein was used for immunostaining. Results: Increased glucose metabolism was observed in the bilateral amygdala after fearconditioning $(P<0.001)$ and in the right posterior insular cortex under extinction retrieval $(P<0.001)$ compared with the control phase. Increased c-Fos expression in the posterior insular cortex under extinction retrieval was positively correlated to the glucose metabolism $(P<$ 0.01). Conclusion: Our results indicated that the amygdala plays a key role in fear memory formation and, most importantly, the insular cortex is related to the retrieval of extinction memory. ${ }^{18}$ F-FDG PET may provide a promising in vivo approach for evaluating exposurebased therapy of PTSD.

Key Words: positron emission tomography (PET); post-traumatic stress disorder (PTSD); fear conditioning; extinction

J Nucl Med 2016; 57:1474-1477

DOI: 10.2967/jnumed.116.173443

\section{$\mathbf{P}$} chiatric disorder, affecting up to $40 \%$ of individuals over lifetime exposure to traumatic events $(1,2)$. Over the past decades, considerable studies have explored how fear memories are encoded in the brain. A neurocircuitry model of PTSD emphasized the importance of the amygdala, as well as its interactions with the ventral/ medial prefrontal cortex (PFC) and hippocampus (3). In accord with this model, initial neuroimaging studies of PTSD provided evidence for exaggerated amygdala responses and attenuated ventral/ medial PFC responses during exposure to reminders of the traumatic event (3). In addition, Pavlovian fear-conditioning studies highlighted the key role of the amygdala in the acquisition and storage

Received Jan. 31, 2016; revision accepted Feb. 18, 2016.

For correspondence or reprints contact: Mei Tian, Department of Nuclear Medicine, The Second Hospital of Zhejiang University School of Medicine, 88 Jiefang Rd., Hangzhou, Zhejiang 310009, China.

E-mail: meitian@zju.edu.cn

${ }^{*}$ Contributed equally to this work.

Published online Mar. 16, 2016.

COPYRIGHT (c) 2016 by the Society of Nuclear Medicine and Molecular Imaging, Inc. of conditioned fear memories $(4,5)$. Electrophysiologic recording and inactivation studies in rats suggest that fear extinction depended on increased neuroactivity in the medial PFC under extinction training $(6,7)$. Furthermore, the amygdala has been found activated during fear acquisition (8) and positively correlated with the severity of PTSD symptoms (9). Failure to recall fear extinction memory is associated with lower activation in the hippocampus and ventral/medial PFC in PTSD patients relative to trauma-exposed healthy subjects (10).

Although exposure-based therapy (conceptually based on fear extinction) has been widely used in the treatment of PTSD (1), its underlying mechanism has not been completely elucidated. Because PET has been increasingly used to characterize neural activities, we hypothesized that ${ }^{18} \mathrm{~F}$-FDG PET could be applied for evaluating cerebral glucose metabolism before and after exposure-based therapy and could provide a potential translational tool for future clinical applications. Thus, the present study aimed to investigate the neurobiologic changes by ${ }^{18} \mathrm{~F}-\mathrm{FDG}$ PET in a rat model of PTSD.

\section{MATERIALS AND METHODS}

\section{Animals}

Male Sprague-Dawley rats ( $n=25$; body weight, $260-280 \mathrm{~g}$ ) were housed under standard laboratory conditions with food and water ad libitum. All animal experiments were performed with the approval of the Institutional Animal Care and Use Committee at Zhejiang University (Protocol ZJU201407-1-02-067).

\section{Behavioral Procedures}

Pavlovian fear-conditioning and extinction procedures were conducted in standard operant chambers (AniLab Software \& Instruments Co., Ltd.) (11). Rats were habituated to handling and to the conditioning chamber for $2 \mathrm{~d}$ before training. Each rat was presented with 10 tones $(30 \mathrm{~s}, 75 \mathrm{~dB})$ only on day 1 (serves as the control phase) and fear-conditioned with 10 sets of tones $(30 \mathrm{~s}, 75 \mathrm{~dB})$ that coterminated with a footshock $(1 \mathrm{~s}$, $0.5 \mathrm{~mA}$ ) on day 2 in the conditioning chamber (Figs. 1A and 1B). Then the rats were moved to the extinction chamber and presented with 20 tones on days 3 and 4 and tested for recall of extinction memory with 10 tones alone on day 5. All the procedures were videotaped, and freezing percentage was calculated as the percentage of time of the total tone duration when the rat remained immobile (frozen).

\section{Image Acquisition and Analysis}

PET imaging studies were done on days 1, 2, and 5 (Fig. 1A). Each rat performed the behavior training for $30 \mathrm{~min}$, and PET images were acquired in the micro-PET R4 scanner (Siemens Medical Solutions) 


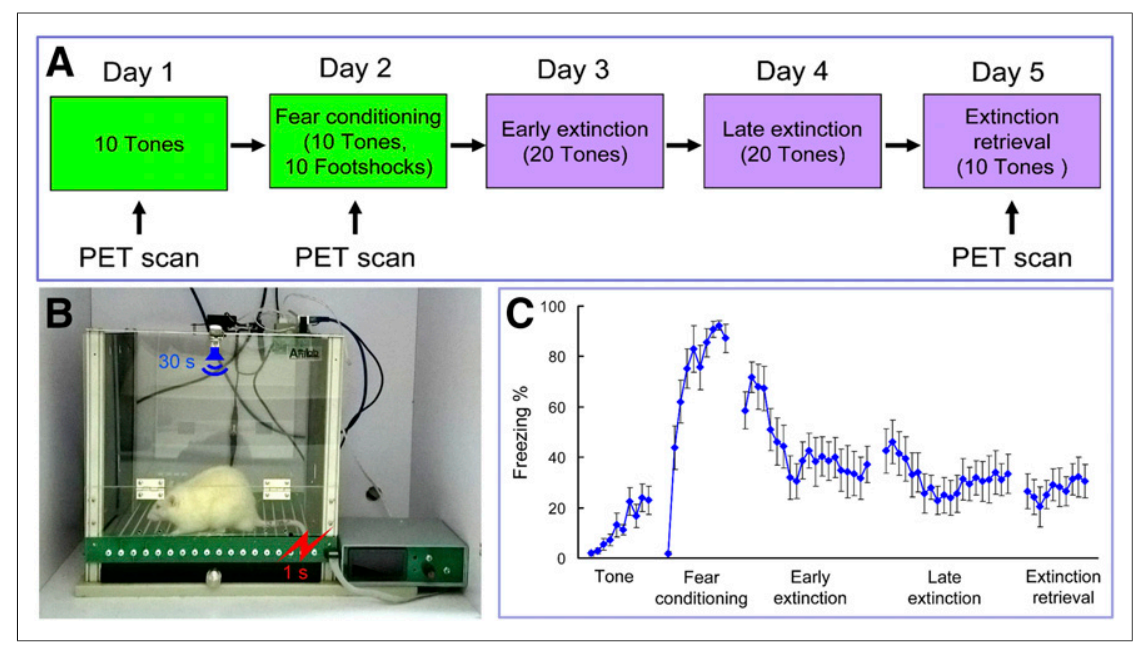

FIGURE 1. Experimental design and behavioral results. (A) Schematic of behavioral paradigm. (B) Rat was fear-conditioned with 30-s tone that coterminated with a footshock. (C) Freezing percentage in different phases. Data are mean \pm SEM $(n=10)$. left primary somatosensory cortex, and left ventroposterior medial (VPM) nucleus of the thalamus $(P<0.001)$ under fear-conditioning compared with the control phase (Table 1; Fig. 2; Supplemental Fig. 1). After extinction retrieval, ${ }^{18} \mathrm{~F}-\mathrm{FDG}$ accumulations were increased $(P<0.001)$ in the right primary visual cortex and right posterior insular cortex but decreased $(P<0.001)$ in a cluster comprising the right orbital cortex, lateral septum, and bilateral bed nucleus of the stria terminalis compared with the control (Table 2; Fig. 3; Supplemental Fig. 2).

After extinction retrieval, c-Fos-positive cells in the posterior insular cortex were significantly increased compared with the fear-conditioning $(P<0.01$, Fig. $4 \mathrm{~A})$ and positively correlated with the ${ }^{18} \mathrm{~F}-\mathrm{FDG}$ accumulation $(P<0.01$, Fig. 4B). at $40 \mathrm{~min}$ after intraperitoneal injection of ${ }^{18} \mathrm{~F}-\mathrm{FDG}(18.5 \mathrm{MBq})$. Images were analyzed by statistical parametric mapping (12).

\section{Immunohistochemistry}

The neuronal activation marker c-Fos was immunostained in the specific brain regions in which increased ${ }^{18} \mathrm{~F}$-FDG accumulations were found. Immunohistological procedures were performed as described previously (13). Slides were incubated with a polyclonal antibody against c-Fos (1:1,000; Santa Cruz Biotechnology) overnight at $4^{\circ} \mathrm{C}$, and c-Fospositive cells were calculated. Data are presented as mean \pm SEM. Differences were considered significant if the $P$ value was less than 0.05 .

\section{RESULTS}

Fear-conditioning induced significant increases of freezing percentage compared with the control tone phase (peak freezing percentage of $87.2 \%$ vs. $23.6 \%$ ) (Fig. 1C). Freezing percentage decreased gradually after the early and late extinction training, and during extinction retrieval freezing percentages were stabilized within a lower range.

${ }^{18} \mathrm{~F}-\mathrm{FDG}$ accumulations were increased in the bilateral amygdala $(P<0.001)$ but decreased in the bilateral secondary motor cortex,

\section{DISCUSSION}

The present study investigated the changes of brain metabolism and neuroactivity during fear-conditioning and extinction retrieval. We found increased glucose metabolism in the bilateral amygdala under fear conditioning and in the right posterior insular cortex after extinction retrieval. Other areas with decreased glucose metabolism were observed, including the bilateral secondary motor cortex, left primary somatosensory cortex, left VPM under fear conditioning, and the lateral septum and bilateral bed nucleus of the stria terminalis after extinction retrieval compared with the control phase. Moreover, overexpression of the neuroactivity marker c-Fos was associated with increased glucose metabolism in the posterior insular cortex after extinction retrieval.

Interpreted in the context of other clinical PET imaging studies, the amygdala is activated during fear acquisition in PTSD patients (8) and is involved in processing fearful faces in healthy subjects (14). Functional MRI revealed exaggerated amygdala responses to fearful faces and that responses were positively correlated with the severity of PTSD symptoms (9). Our finding of activated bilateral amygdala under fear-conditioning is consistent with the above listed references and confirmed our hypothesis of using PET

TABLE 1

Significant Glucose Metabolic Changes Under Fear Conditioning (Control vs. Fear-Conditioning)

\begin{tabular}{|c|c|c|c|c|c|c|}
\hline \multirow[b]{2}{*}{ Region } & \multicolumn{3}{|c|}{ Coordinate (mm) } & \multicolumn{3}{|c|}{ Cluster level } \\
\hline & $x$ & $y$ & $z$ & $t$ value & z score & $P_{\text {uncorrected }}$ \\
\hline \multicolumn{7}{|l|}{ Increased } \\
\hline Left amygdala & -5 & 8 & -3 & 4.65 & 3.56 & $<0.001$ \\
\hline Right amygdala & 5 & 8 & -3 & 4.32 & 3.39 & $<0.001$ \\
\hline \multicolumn{7}{|l|}{ Decreased } \\
\hline Right secondary motor cortex & 2 & 2 & 5 & 5.90 & 4.11 & $<0.001$ \\
\hline Left secondary motor cortex & -2 & 2 & 4 & 5.35 & 3.88 & $<0.001$ \\
\hline Left primary somatosensory cortex (forelimb and jaw area) & -5 & 2 & 2 & 5.15 & 3.80 & $<0.001$ \\
\hline Left VPM nucleus of the thalamus & -3 & 6 & -4 & 4.22 & 3.33 & $<0.001$ \\
\hline
\end{tabular}




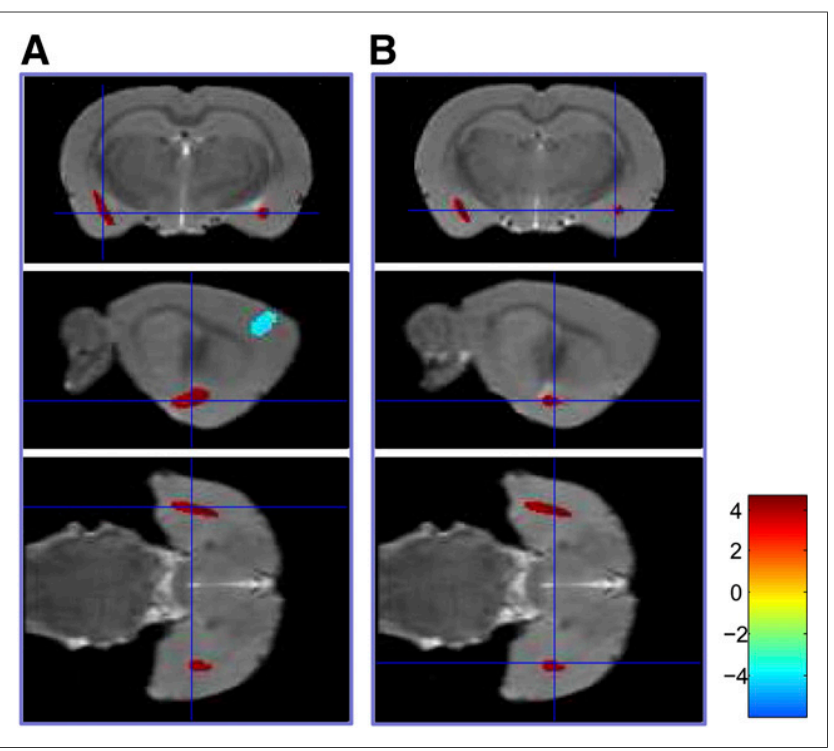

FIGURE 2. Coronal, sagittal, and transverse images demonstrated increased glucose metabolism in left (A) and right (B) amygdala under fear-conditioning $(n=8, P<0.001)$.

technology to investigate neurofunctional changes in the rat model of PTSD. Interestingly, we observed decreased glucose metabolism in the bilateral secondary motor cortex, left primary somatosensory cortex (forelimb and jaw area), and left VPM under fearconditioning. Because VPM is a somatosensory relay station that relays input sensory information from individual whiskers and projects to the somatosensory cortex (15) in rats, decreased glucose metabolism in this region was associated with increased freezings and reduced exploring behaviors during fear-conditioning (15).

The most important finding of the present study was the increased glucose metabolism and activated c-Fos expression in the right posterior insular cortex after extinction retrieval. To our knowledge, this is the first study to show that the posterior insular cortex is involved in the retrieval of fear extinction memory in rodents. The insular cortex is important for the acquisition and extinction of conditioned taste aversion (16), and the extinction rate is positively correlated with c-fos messenger RNA expression in rats (17). Bilateral inhibition of the posterior insular cortex during
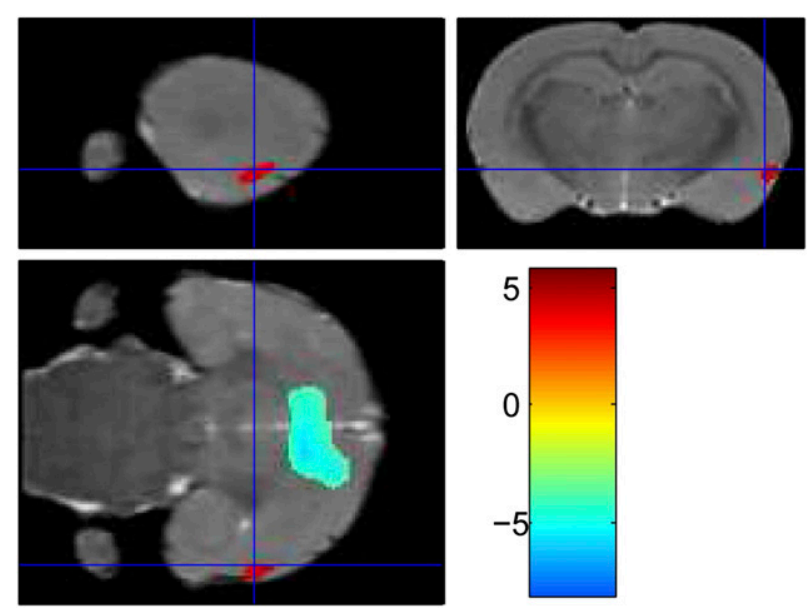

FIGURE 3. Sagittal, coronal, and transverse images demonstrated increased glucose metabolism in right posterior insular cortex under extinction retrieval $(n=8, P<0.001)$.

stress exposure prevented the stress-mitigating effect of safety signals (18). The posterior insular cortex projects strongly to GABAergic (GABA is $\gamma$-aminobutyric acid) neurons in the lateral subdivision of central amygdala $(\mathrm{CeL})$ in rats (19), which serves as a switch capable of orchestrating the activity of projection neurons in the medial subdivision of central amygdala (CeM) and consequently regulates conditioned fear responses $(20,21)$. Therefore, we speculated that inhibitory $\mathrm{CeL}$ neurons were activated by projection neurons in the posterior insular cortex during extinction retrieval, leading to inhibition of CeM projection neurons and thus suppression of fear responses.

The involvement of the amygdala and insular cortex has also been demonstrated in previous human studies. Especially, repeated exposure to traumatic memory (used as an exposure-based treatment) could increase functional connectivities between right amygdala and bilateral anterior insular cortex, as well as between left amygdala and right anterior insular cortex in PTSD patients (22). Repeated presentation of negative images to healthy subjects could increase bilateral posterior insular cortex activity and was associated with increased functional connectivity between the left posterior insular cortex and amygdala (23). In addition, a smaller insular

TABLE 2

Significant Glucose Metabolic Changes Under Extinction Retrieval (Control vs. Extinction Retrieval)

\begin{tabular}{|c|c|c|c|c|c|c|}
\hline \multirow[b]{2}{*}{ Region } & \multicolumn{3}{|c|}{ Coordinate (mm) } & \multicolumn{3}{|c|}{ Cluster level } \\
\hline & $x$ & $y$ & $z$ & $t$ value & $z$ score & $P_{\text {uncorrected }}$ \\
\hline \multicolumn{7}{|l|}{ Increased } \\
\hline Right primary visual cortex & 3 & 2 & -6 & 5.75 & 4.05 & $<0.001$ \\
\hline Right insular cortex & 6 & 7 & -2 & 4.15 & 3.30 & $<0.001$ \\
\hline \multicolumn{7}{|l|}{ Decreased } \\
\hline Right orbital cortex & 1 & 5 & 4 & 8.00 & 4.83 & $<0.001$ \\
\hline Left lateral septum & -1 & 6 & 1 & 8.00 & 4.83 & $<0.001$ \\
\hline Right lateral septum & 1 & 6 & 1 & 8.00 & 4.83 & $<0.001$ \\
\hline Right bed nucleus of the stria terminalis & -1 & 7 & 0 & 5.74 & 4.05 & $<0.001$ \\
\hline Left bed nucleus of the stria terminalis & 1 & 7 & 0 & 5.56 & 3.98 & $<0.001$ \\
\hline
\end{tabular}




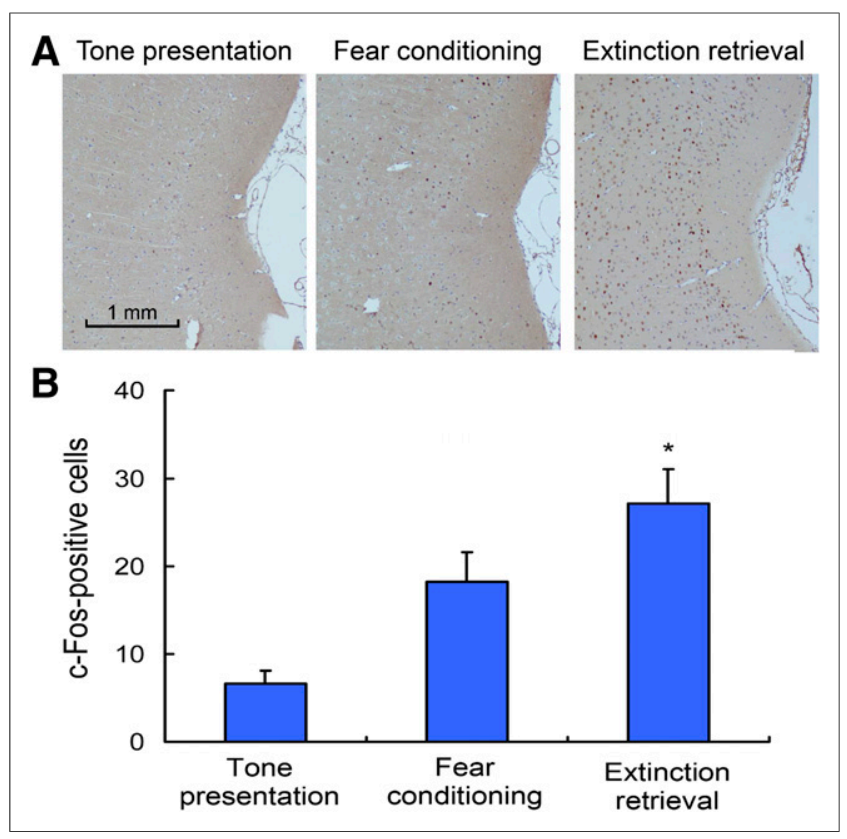

FIGURE 4. Expression of c-Fos in posterior insular cortex. (A) Representative photomicrographs of immunolabeled c-Fos neurons in tone presentation, fear-conditioning, and extinction retrieval phases. (B) Quantification of c-Fos-positive neurons in posterior insular cortex $(n=5$ in each phase, ${ }^{\star} P<0.01$ ).

cortex was found in PTSD patients than trauma-exposed healthy subjects, indicating deficient extinction processes and an uncontrollable state of fear $(24,25)$. Consistent with previous studies, our PET imaging findings combined with immunohistological data indicated that the insular cortex plays a critical role in the retrieval of extinction memory.

\section{CONCLUSION}

Our results support a key role for the amygdala in fear memory formation. The PET imaging findings combined with immunohistological data provide compelling evidence that the posterior insular cortex is involved in the retrieval of extinction memory. PET imaging of fear circuitry in animal models may provide a valuable translational approach to better characterize pathophysiologic mechanisms of PTSD. Future studies are required to better delineate the contribution of the insular cortex in extinction retrieval and its functional connectivity with other brain regions.

\section{DISCLOSURE}

The costs of publication of this article were defrayed in part by the payment of page charges. Therefore, and solely to indicate this fact, this article is hereby marked "advertisement" in accordance with 18 USC section 1734. This work is partly sponsored by grants from the National Natural Science Foundation of China (81271601), National Science Fund for Distinguished Young Scholars (81425015), Zhejiang Provincial Natural Science Foundation of China (LR13H180001), Specialized Research Fund for the Doctoral Program of Higher Education (20130101110015), Zhejiang Province, and the National Health Department joint construction project
(WKJ2013-2-016). No other potential conflict of interest relevant to this article was reported.

\section{REFERENCES}

1. Powers MB, Halpern JM, Ferenschak MP, Gillihan SJ, Foa EB. A meta-analytic review of prolonged exposure for posttraumatic stress disorder. Clin Psychol Rev. 2010;30:635-641.

2. Ressler KJ, Mercer KB, Bradley B, et al. Post-traumatic stress disorder is associated with PACAP and the PAC1 receptor. Nature. 2011;470:492-497.

3. Rauch SL, Shin LM, Phelps EA. Neurocircuitry models of posttraumatic stress disorder and extinction: human neuroimaging research-past, present, and future. Biol Psychiatry. 2006;60:376-382.

4. Pitman RK, Rasmusson AM, Koenen KC, et al. Biological studies of post-traumatic stress disorder. Nat Rev Neurosci. 2012;13:769-787.

5. Parsons RG, Ressler KJ. Implications of memory modulation for post-traumatic stress and fear disorders. Nat Neurosci. 2013;16:146-153.

6. Milad MR, Quirk GJ. Neurons in medial prefrontal cortex signal memory for fear extinction. Nature. 2002;420:70-74.

7. Santini E, Ge H, Ren K, Pena de Ortiz S, Quirk GJ. Consolidation of fear extinction requires protein synthesis in the medial prefrontal cortex. $J$ Neurosci. 2004;24:5704-5710.

8. Bremner JD, Vermetten E, Schmahl C, et al. Positron emission tomographic imaging of neural correlates of a fear acquisition and extinction paradigm in women with childhood sexual-abuse-related post-traumatic stress disorder. Psychol Med. 2005;35:791-806.

9. Armony JL, Corbo V, Clement MH, Brunet A. Amygdala response in patients with acute PTSD to masked and unmasked emotional facial expressions. Am J Psychiatry. 2005;162:1961-1963.

10. Milad MR, Pitman RK, Ellis CB, et al. Neurobiological basis of failure to recall extinction memory in posttraumatic stress disorder. Biol Psychiatry. 2009;66:1075-1082.

11. Burgos-Robles A, Vidal-Gonzalez I, Santini E, Quirk GJ. Consolidation of fear extinction requires NMDA receptor-dependent bursting in the ventromedial prefrontal cortex. Neuron. 2007;53:871-880.

12. $\mathrm{Xi} \mathrm{W}, \mathrm{Su} \mathrm{D}$, Nie B, et al. ${ }^{18} \mathrm{~F}$-FDG PET study reveals brain functional changes during attention in rats. $J$ Nucl Med. 2013;54:1969-1973.

13. Orsini CA, Kim JH, Knapska E, Maren S. Hippocampal and prefrontal projections to the basal amygdala mediate contextual regulation of fear after extinction. J Neurosci. 2011;31:17269-17277.

14. Morris JS, Frith CD, Perrett DI, et al. A differential neural response in the human amygdala to fearful and happy facial expressions. Nature. 1996;383:812-815.

15. Petersen CC. The functional organization of the barrel cortex. Neuron. 2007;56: 339-355.

16. Berman DE, Dudai Y. Memory extinction, learning anew, and learning the new: dissociations in the molecular machinery of learning in cortex. Science. 2001;291:2417-2419.

17. Hadamitzky M, Bosche K, Engler A, Schedlowski M, Engler H. Extinction of conditioned taste aversion is related to the aversion strength and associated with c-fos expression in the insular cortex. Neuroscience. 2015;303:34-41.

18. Christianson JP, Jennings JH, Ragole T, et al. Safety signals mitigate the consequences of uncontrollable stress via a circuit involving the sensory insular cortex and bed nucleus of the stria terminalis. Biol Psychiatry. 2011;70:458-464.

19. McDonald AJ, Shammah-Lagnado SJ, Shi C, Davis M. Cortical afferents to the extended amygdala. Ann N Y Acad Sci. 1999;877:309-338.

20. Ciocchi S, Herry C, Grenier F, et al. Encoding of conditioned fear in central amygdala inhibitory circuits. Nature. 2010;468:277-282.

21. Haubensak W, Kunwar PS, Cai H, et al. Genetic dissection of an amygdala microcircuit that gates conditioned fear. Nature. 2010;468:270-276.

22. Cisler JM, Steele JS, Lenow JK, et al. Functional reorganization of neural networks during repeated exposure to the traumatic memory in posttraumatic stress disorder: an exploratory fMRI study. J Psychiatr Res. 2014;48:47-55.

23. Denny BT, Fan J, Liu X, et al. Insula-amygdala functional connectivity is correlated with habituation to repeated negative images. Soc Cogn Affect Neurosci. 2014;9:1660-1667.

24. Kasai K, Yamasue H, Gilbertson MW, Shenton ME, Rauch SL, Pitman RK. Evidence for acquired pregenual anterior cingulate gray matter loss from a twin study of combat-related posttraumatic stress disorder. Biol Psychiatry. 2008; 63:550-556.

25. Chen S, Xia W, Li L, et al. Gray matter density reduction in the insula in fire survivors with posttraumatic stress disorder: a voxel-based morphometric study. Psychiatry Res. 2006;146:65-72. 